\title{
A avaliabilidade dos Núcleos de Apoio à Saúde da Família (Nasf)
}

\author{
The evaluability assessment of the Family Health Support Centers \\ (Nasf)
}

Patrícia Caroline lacabo Correia', Patrícia Martins Goulart², Juarez Pereira Furtado $\mathbf{3}$

RESUMO Os Núcleos de Apoio à Saúde da Família (Nasf) vêm se constituindo enquanto objeto de indagações e pesquisas. Com base em revisão de literatura e no discurso oficial expresso nos documentos do Ministério da Saúde, realizou-se o presente estudo de sua avaliabilidade, apontando algumas das estratégias de avaliação mais adequadas à iniciativa. A proposta Nasf se mostrou, portanto, avaliável, e a avaliação de sua lógica e estratégia, abordando sua concepção e hipóteses teóricas, ganha relevância no atual estágio em que a iniciativa se encontra.

PALAVRAS-CHAVE Avaliação em saúde. Saúde da família. Atenção Primária à Saúde.

ABSTRACT The Family Health Support Centers (Nasf) have become an object of inquiry and research. Based on a review of literature and official speech expressed in the documents of the Ministry of Health, a study of its evaluability was conducted, pointing out some assessment strategies more appropriate to the initiative. Therefore, the Nasf proposal proved to be evaluable, and the evaluation of its logic and strategy, approaching its conception and theoretical hypotheses, gains relevance in its current stage, in which the initiative is found.

KEYWORDS Health evaluation. Family health. Primary Health Care.

1 Universidade Federal de São Paulo (Unifesp), Programa de Pós-

Graduação em Alimentos, Nutrição e Saúde - Santos (SP), Brasil.

patricia.iacabo@gmail.com

2 Universidade Federal de São Paulo (Unifesp), Departamento de Políticas Públicas e Saúde Coletiva Santos (SP), Brasil. pmg.correio@gmail.com

3 Universidade Federal de São Paulo (Unifesp), Departamento de Políticas Públicas e Saúde Coletiva Santos (SP), Brasil.

juarezpfurtado@hotmail.com 


\section{Introdução}

O Núcleo de Apoio à Saúde da Família (Nasf) configura-se como uma política instituída pelo Ministério da Saúde (MS), por meio da Portaria Gabinete do Ministro/Ministério da Saúde (GM/MS) n ${ }^{\circ} 154$, de 24 de janeiro de 2008 (BRASIL, 2008). Constituído por equipes de profissionais de distintas áreas do conhecimento, que atuam em articulação com as Equipes de Saúde da Família (EqSF) e as equipes da Atenção Básica (AB) para populações específicas - consultório na rua, equipes ribeirinhas e fluviais, entre outras -, em territórios definidos, este arranjo tem por objetivo ampliar a resolutividade da $A B$ (BRASIL, 2011).
Indagações sobre parâmetros e atribuições essenciais do Nasf, em geral, e dos papéis de seus respectivos profissionais, em particular, podem ser identificadas nas práticas cotidianas desses núcleos e também em textos científicos (SAMPAIO ET AL., 2012), indicando uma possível insuficiência dos referenciais e direcionamentos estabelecidos pelo MS (BRASIL, 2014) para guiar a iniciativa. O surgimento de questões sobre o que e como desenvolver as atividades relativas ao Nasf é sincrônico à ampliação numérica desses núcleos. Vale ressaltar que este total mais do que duplicou nos últimos três anos, indo de 1.983 equipes, em janeiro de 2013, para 4.462, em janeiro de 2016, sendo considerados os três tipos existentes (gráfico 1).

Gráfico 1. Evolução da implantação do Nasf no Brasil, de 2008 a 2016. Competência Sistema de Cadastro Nacional de Estabelecimentos de Saúde (SCNES): Fev./2016.

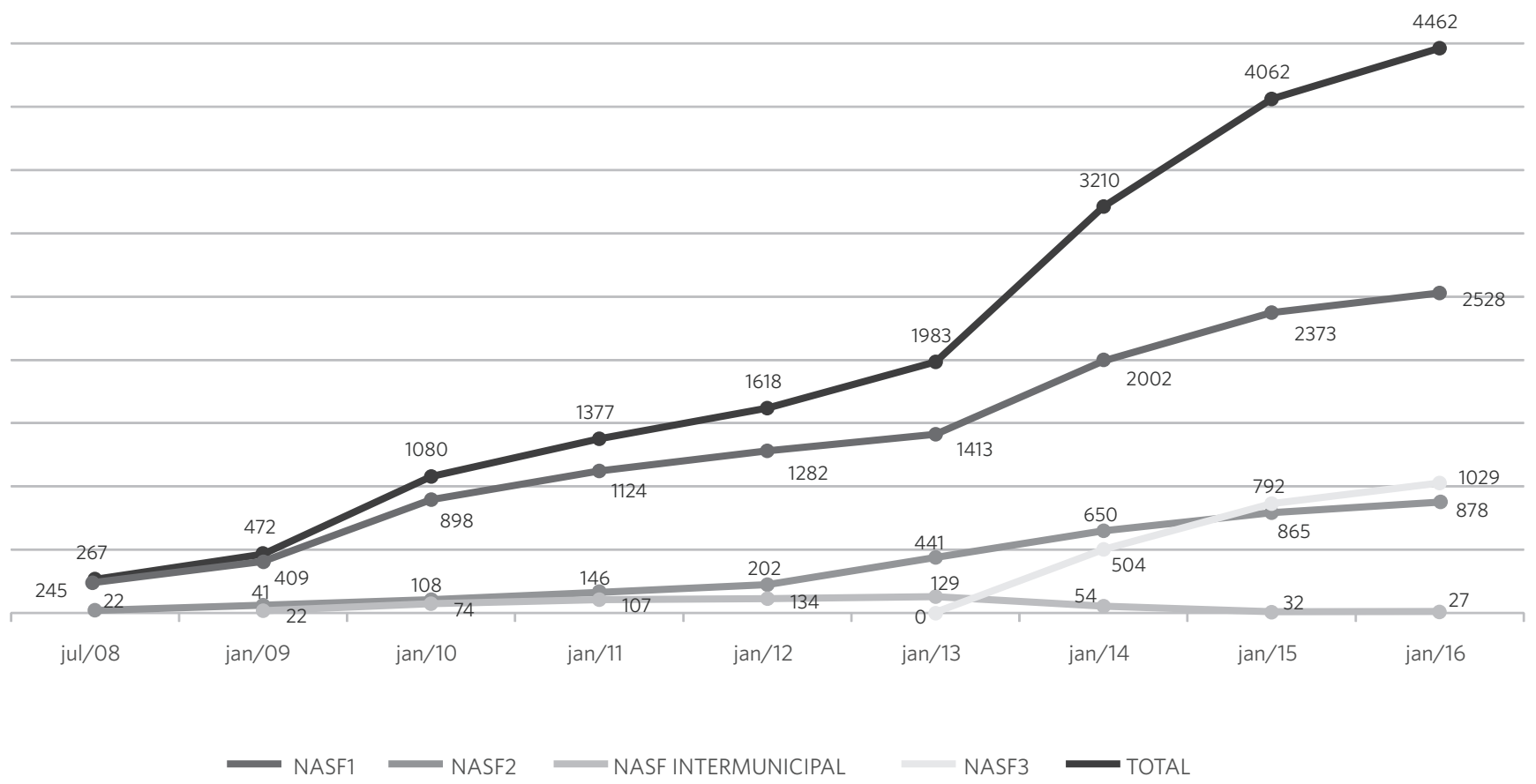


A potência indutora do MS pode ser ilustrada pela inflexão positiva das curvas de crescimento do Nasf (gráfico 1), logo após o lançamento da Portaria GM/MS n ${ }^{\circ} 2.488$, de 21 de outubro de 2011 (BRASIL, 2011), mais flexível do que a anterior (BRASIL, 2008). No entanto, a capacidade do MS de orientar e esclarecer as equipes sobre as estratégias de implantação e condução dos Nasf, ainda que estas sejam detalhadas em suas respectivas portarias e publicações ministeriais, no plano de concepções e operacionalização (BRASIL, 2008, 2009, 2011, 2012, 2014), parece não subsidiar a efetiva condução dos núcleos nos milhares de municípios brasileiros - sobretudo, se consideradas as dificuldades de coordenação federativa em saúde e de organização regionalizada do Sistema Único de Saúde (SUS), no plano estadual (MACHADO ET AL., 2014). Como resultado, a proposta de Nasf frequentemente é objeto de dúvidas, questões, dissensos e interpretações diversas ao alcançar o plano municipal, tomando distintas configurações conforme a localidade em que está implantada.

Sabe-se que uma das formas do SUS desenvolver suas políticas de saúde se dá de maneira centralizada, por meio de diretrizes, portarias e fomentos financeiros. Esta forma de atuação gera uma verticalização que atravessa as diferentes instâncias do próprio SUS - MS, Secretaria Estadual de Saúde, Secretaria Municipal de Saúde, Rede de Atenção à Saúde (RAS) e serviços. Como consequência, alguns modelos de gestão e operacionalização de certas políticas tendem a uma normatização burocrática e descontextualizada, calcada em metas e procedimentos destituídos de legitimidade e/ ou pertinência, na base do sistema de saúde (FURTADO; ONOCKO-CAMPOS, 2005).

Iniciativas de avaliação dos Nasf se beneficiariam ao sistematizarem as proposições governamentais e as confrontarem com o conjunto de questões surgidas da implementação da proposta e evidenciadas diretamente pelos trabalhadores e/ou por meio de diferentes pesquisas. Assim sendo, considera-se a importância do estudo da avaliabilidade dos Nasf para subsidiar suas futuras avaliações. A avaliabilidade, ou pré-avaliação, foi originalmente formulada por Joseph Wholey nos anos 1970 (WHOLEY, 1987). Para este autor, a avaliabilidade permite verificar a viabilidade de processos avaliativos e aumentar as chances de uso de futuras avaliações, na medida em que explora as expectativas e necessidades dos grupos de interesse envolvidos e o grau de organização e implementação da iniciativa. A avaliabilidade permite mapear desacordos entre os grupos de interesse ou destes com os propósitos de um programa ou serviço, sendo especialmente útil na definição de quais aspectos do programa necessitariam ser avaliados (MENDES; BEZERRA; DUBEUX, 2010). Os estudos de avaliabilidade vêm sendo utilizados mais recentemente no Brasil, dirigidos a alguns programas na área da saúde (MEDEIROS ET AL., 2010; PADILHA; OLIVEIRA; FIGUEIRÓ, 2015; OLIVEIRA ET AL., 2015).

Por meio deste estudo de avaliabilidade, buscou-se evidenciar possíveis insuficiências e inconsistências presentes na concepção e orientação dos Nasf, do ponto de vista de seus implementadores e pesquisadores, considerando-se as principais questões que vêm sendo apontadas. Deste modo, seria possível subsidiar consensos em torno de concepções essenciais dos Nasf, bem como explicitar divergências e identificar conhecimentos prioritários a serem levantados sobre eles, ampliando a potencialidade e a adequação de futuras avaliações. Estudos de avaliabilidade preveem a sistematização gráfica da iniciativa e a legitimação dessa representação junto aos grupos de interesse; analisam a clareza e a pertinência da proposta, bem como o grau de implementação e, finalmente, o estabelecimento de possíveis questões avaliativas. Além disso, em alguns casos, a definição de indicadores de avaliação, sempre em colaboração com esses mesmos grupos de agentes, de alguma forma envolvidos com um dado programa ou serviçO (WHOLEY, 1987; MENDES; BEZERRA; DUBEUX, 2010). 


\section{Métodos}

No presente estudo, os grupos de interesse serão constituídos por trabalhadores e pesquisadores, a partir do material por eles publicado e abordado por meio de revisão de artigos em bases indexadas, fontes que serão usadas para analisar a proposta e identificar questões avaliativas. A proposta de Nasf foi sistematizada com base no discurso oficial expresso em documentos do MS, elaborando-se o seu modelo lógico. Esse percurso permitirá analisar convergências e divergências entre o campo burocrático e o campo científico, no que tange a esses núcleos, determinando sua avaliabilidade e possíveis questões para eventuais avaliações (MENDES; BEZERRA; DUBEUX, 2010; WHOLEY, 1987).

A busca na literatura, realizada em fevereiro de 2016, abrangeu o período entre 2008 (ano da publicação da primeira portaria referente aos Nasf) e 2016. Foram consideradas as orientações da Cochrane Collaboration (HIGGINS; GREEN, 2011) nas bases Scientific Electronic Library Online (SciELO), Literatura Latino-Americana e do Caribe em Ciências da Saúde (Lilacs), PubMed, Scopus e Web of Science. Dada a ausência de descritor, foram utilizados os seguintes termos livres, nos títulos e resumos dos artigos: 'Núcleo de Apoio à Saúde da Família'; 'Family Health Support Center'; 'Support team for family health strategy'; e
'Nasf'. Considerou-se que a presença dessa expressão no título e/ou no resumo seria indício de uma abordagem central do tema.

A condução da revisão seguiu os seguintes passos: 1) busca na literatura; 2) leitura de títulos e resumos de todos os artigos, de forma independente, por duas pesquisadoras; 3) exclusão de textos em outros formatos que não os de artigo, não disponíveis em português ou inglês, artigos duplicados ou que não abordavam especificamente a temática do estudo; 4) busca na literatura cinzenta; 5) consenso sobre a seleção dos artigos; 6) leitura, na íntegra, dos artigos selecionados; 7) fichamento de todos os artigos; 8) consenso para dirimir dúvidas entre os dados coletados e unificá-los para análise e interpretação dos artigos; 9) identificação de concepção, práticas e experiências na implantação dos Nasf. Entre as 278 publicações (85 na SciELO, 93 na Lilacs, 30 na PubMed, 37 na Scopus e 33 na Web of Science), 123 foram excluídas por estarem duplicadas e 120 foram descartadas por não abordarem a temática em estudo. Um artigo da literatura cinzenta foi acrescentado, totalizando 36 artigos (quadro 1).

O presente estudo possui limitações, entre elas, aquela gerada pela inexistência de descritores para o levantamento dos artigos. Entretanto, observou-se a saturação dos dados nos artigos selecionados, indício de que a revisão evidenciou o estado da arte dessa problemática. 


\begin{tabular}{|c|c|c|c|}
\hline Autores & Título & Revista & Ano \\
\hline Barbosa et al. & $\begin{array}{l}\text { Experiência da fisioterapia no Núcleo de Apoio à Saúde da Família em Governador } \\
\text { Valadares, MG }\end{array}$ & Fisioterapia em Movimento & 2010 \\
\hline Lancman e Barros & $\begin{array}{l}\text { Estratégia de Saúde da Família (ESF), Núcleo de Apoio à Saúde da Família (Nasf) e } \\
\text { terapia ocupacional: problematizando as interfaces }\end{array}$ & $\begin{array}{l}\text { Rev. Ter. Ocup. Univ. São } \\
\text { Paulo }\end{array}$ & 2011 \\
\hline Souza e Loch & $\begin{array}{l}\text { Intervenção do profissional de educação física nos Núcleos de Apoio à Saúde da } \\
\text { Família em municípios do norte do Paraná }\end{array}$ & Rev. Bras. Ativ. Fís. Saúde & 2011 \\
\hline Dibai Filho e Aveiro & $\begin{array}{l}\text { Atuação dos fisioterapeutas dos Núcleos de Apoio à Saúde da Família entre idosos } \\
\text { do município de Arapiraca-AL, Brasil }\end{array}$ & $\begin{array}{l}\text { Rev. Bras. Promoç. Saúde } \\
\text { (Impr.) }\end{array}$ & 2012 \\
\hline Reis et al. & $\begin{array}{l}\text { Perfil de atendimento de um Núcleo de Apoio à Saúde da Família na área de reabilita- } \\
\text { ção, Município de Belo Horizonte, Estado de Minas Gerais, Brasil, } 2009\end{array}$ & $\begin{array}{l}\text { Epidemiologia e Serviços } \\
\text { de Saúde }\end{array}$ & 2012 \\
\hline Sampaio et al. & O Nasf como dispositivo da gestão: limites e possibilidades & $\begin{array}{l}\text { Revista Brasileira de Ciên- } \\
\text { cias da Saúde }\end{array}$ & 2012 \\
\hline $\begin{array}{l}\text { Vannucchi e Car- } \\
\text { neiro Junior }\end{array}$ & $\begin{array}{l}\text { Modelos tecnoassistenciais e atuação do psiquiatra no campo da atenção primária à } \\
\text { saúde no contexto atual do Sistema Único de Saúde, Brasil }\end{array}$ & $\begin{array}{l}\text { Physis: Revista de Saúde } \\
\text { Coletiva }\end{array}$ & 2012 \\
\hline Anjos et al. & $\begin{array}{l}\text { Perspectivas e desafios do Núcleo de Apoio à Saúde da Família quanto às práticas em } \\
\text { saúde }\end{array}$ & Saúde em Debate & 2013 \\
\hline $\begin{array}{l}\text { Araújo e Galim- } \\
\text { bertti }\end{array}$ & A colaboração interprofissional na Estratégia Saúde da Família & Psicologia \& Sociedade & 2013 \\
\hline Azevedo e Kind & Psicologia nos Núcleos de Apoio à Saúde da Família em Belo Horizonte & $\begin{array}{l}\text { Psicologia: Ciência e Pro- } \\
\text { fissão }\end{array}$ & 2013 \\
\hline Costa et al. & $\begin{array}{l}\text { A prática do fonoaudiólogo nos Núcleos de Apoio à Saúde da Família em municípios } \\
\text { paraibanos }\end{array}$ & CoDAS & 2013 \\
\hline Falci e Belisário & $\begin{array}{l}\text { A inserção do profissional de educação física na atenção primária à saúde e os desa- } \\
\text { fios em sua formação }\end{array}$ & $\begin{array}{l}\text { Interface - Comunicação, } \\
\text { Saúde, Educação }\end{array}$ & 2013 \\
\hline Fernandes et al. & $\begin{array}{l}\text { Análise das atribuições dos fonoaudiólogos do Nasf em municípios da região metro- } \\
\text { politana do Recife }\end{array}$ & Revista CEFAC & 2013 \\
\hline Lancman et al. & Estudo do trabalho e do trabalhar no Núcleo de Apoio à Saúde da Família & Revista de Saúde Pública & 2013 \\
\hline Leite et al. & A inserção da Psicologia nos Núcleos de Apoio à Saúde da Família & $\begin{array}{l}\text { Physis: Revista de Saúde } \\
\text { Coletiva }\end{array}$ & 2013 \\
\hline Pasquim e Arruda & $\begin{array}{l}\text { Núcleo de Apoio à Saúde da Família: revisão narrativa sobre o apoio matricial na } \\
\text { atenção básica }\end{array}$ & Corpus et Scientia & 2013 \\
\hline Reisa e Vieira & $\begin{array}{l}\text { Perspectivas dos terapeutas ocupacionais sobre sua inserção nos Núcleos de Apoio à } \\
\text { Saúde da Família (Nasf) de Fortaleza, CE }\end{array}$ & Cad. Ter. Ocup. UFSCar & 2013 \\
\hline Andrade et al. & Avaliação das ações da fonoaudiologia no Nasf da cidade do Recife & $\begin{array}{l}\text { Audiology - Communica- } \\
\text { tion Research }\end{array}$ & 2014 \\
\hline Bonaldi e Ribeiro & $\begin{array}{l}\text { Núcleo de Apoio à Saúde da Família: as ações de promoção da saúde no cenário da } \\
\text { Estratégia Saúde da Família }\end{array}$ & Revista de APS & 2014 \\
\hline Costa et al. & $\begin{array}{l}\text { Resolubilidade do cuidado na atenção primária: articulação multiprofissional e rede } \\
\text { de serviços }\end{array}$ & Saúde em Debate & 2014 \\
\hline Ferro et al. & $\begin{array}{l}\text { Interdisciplinaridade e intersetorialidade na Estratégia Saúde da Família e no Núcleo } \\
\text { de Apoio à Saúde da Família: potencialidades e desafios }\end{array}$ & Mundo Saúde (Impr.) & 2014 \\
\hline Gomes et al. & $\begin{array}{l}\text { Characteristics of physical activity programs in the Brazilian primary health care } \\
\text { system }\end{array}$ & Cadernos de Saúde Pública & 2014 \\
\hline Hori e Nascimento & $\begin{array}{l}\text { O Projeto Terapêutico Singular e as práticas de saúde mental nos Núcleos de Apoio à } \\
\text { Saúde da Família (Nasf) em Guarulhos (SP), Brasil }\end{array}$ & Ciência \& Saúde Coletiva & 2014 \\
\hline
\end{tabular}




\begin{tabular}{|c|c|c|c|}
\hline Leite et al. & Qualidade de vida no trabalho de profissionais do Nasf no município de São Paulo & $\begin{array}{l}\text { Physis: Revista de Saúde } \\
\text { Coletiva }\end{array}$ & 2014 \\
\hline $\begin{array}{l}\text { Molini-Avejonas } \\
\text { et al. }\end{array}$ & Inserção e atuação da Fonoaudiologia nos Núcleos de Apoio à Saúde da Família & CODAS & 2014 \\
\hline Moura e Luzio & $\begin{array}{l}\text { O apoio institucional como uma das faces da função apoio no Núcleo de Apoio à } \\
\text { Saúde da Família (Nasf): para além das diretrizes }\end{array}$ & $\begin{array}{l}\text { Interface - Comunicação, } \\
\text { Saúde, Educação }\end{array}$ & 2014 \\
\hline Rodrigues e Bosi & O lugar do nutricionista nos Núcleos de Apoio à Saúde da Família & Revista de Nutrição & 2014 \\
\hline Silva et al. & $\begin{array}{l}\text { Atenção Básica em Alagoas: expansão da Estratégia Saúde da Família, do Nasf e do } \\
\text { componente alimentação/nutrição }\end{array}$ & Saúde em Debate & 2014 \\
\hline Aguiar e Costa & Formação e atuação de nutricionistas dos Núcleos de Apoio à Saúde da Família & Revista de Nutrição & 2015 \\
\hline Barros et al. & $\begin{array}{l}\text { Estratégia do apoio matricial: a experiência de duas equipes do Núcleo de Apoio à } \\
\text { Saúde da Família (Nasf) da cidade de São Paulo, Brasil }\end{array}$ & Ciência \& Saúde Coletiva & 2015 \\
\hline Cela e Oliveira & O psicólogo no Núcleo de Apoio à Saúde da Família: articulação de saberes e ações & Estud. Psicol. (Natal) & 2015 \\
\hline Gonçalves et al. & Estudo do trabalho em Núcleos de Apoio à Saúde da Família (Nasf), São Paulo, Brasil & $\begin{array}{l}\text { Revista Brasileira de Saúde } \\
\text { Ocupacional }\end{array}$ & 2015 \\
\hline Matuda et al. & $\begin{array}{l}\text { Colaboração interprofissional na Estratégia Saúde da Família: implicações para a } \\
\text { produção do cuidado e a gestão do trabalho }\end{array}$ & Ciência \& Saúde Coletiva & 2015 \\
\hline Ribeiro et al. & $\begin{array}{l}\text { Representações sociais de profissionais de Núcleos de Apoio à Saúde da Família } \\
\text { sobre interdisciplinaridade }\end{array}$ & Trabalho, Educação e Saúde & 2015 \\
\hline Santos et al. & $\begin{array}{l}\text { The work of physical education professionals in Family Health Support Centers } \\
\text { (Nasf): a national survey }\end{array}$ & $\begin{array}{l}\text { Rev. Bras. Cineantropom. } \\
\text { Desemp. Hum. }\end{array}$ & 2015 \\
\hline Sousa et al. & Entre o especialismo e o apoio: psicólogos no Núcleo de Apoio à Saúde da Família & Psicologia USP & 2015 \\
\hline
\end{tabular}

Fonte: Elaboração própria.

Os documentos oficiais consultados para a construção do modelo lógico foram as seguintes portarias: de criação do Nasf, de aprovação da Política Nacional de Atenção Básica e de redefinição dos parâmetros de vinculação dos Nasf ( ${ }^{\circ} 154 / 2008, n^{\circ} 2.488 / 2011$ e $\mathrm{n}^{\mathrm{o}} 3.124 / 2012$, respectivamente). Isso, além dos 'Cadernos de Atenção Básica' no 27, que apresentam as diretrizes do núcleo, e $\mathrm{n}^{\circ} 39$, que apresentam ferramentas para a gestão e para o trabalho do Nasf (BRASIL, 2008, 2009, 2011, 2012, 2014).

\section{Resultados e discussão}

Os resultados serão apresentados e discutidos em subtópicos elencados a partir da sistematização da proposta do Nasf em um modelo lógico e da exploração da revisão de literatura.

\section{A proposta oficial para os Nasf}

Com base nos documentos oficiais, foi elaborada uma representação gráfica dos Nasf (figura 1), explicitando recursos, atividades, resultados e impacto pretendidos com a iniciativa, conforme discutido na literatura sobre a composição dos chamados modelos lógicos para a avaliação de programas e serviços propostos por Suchman (1967) nos anos 1960, e que hoje apresenta relativo consenso entre avaliadores, sobre sua utilidade em processos avaliativos e de avaliabilidade (FURTADO, 2012; CHAMPAGNE ET AL., 2011B). 
Figura 1. Modelo lógico do Nasf formulado com base em documentos do Ministério da Saúde. Santos, São Paulo, 2016

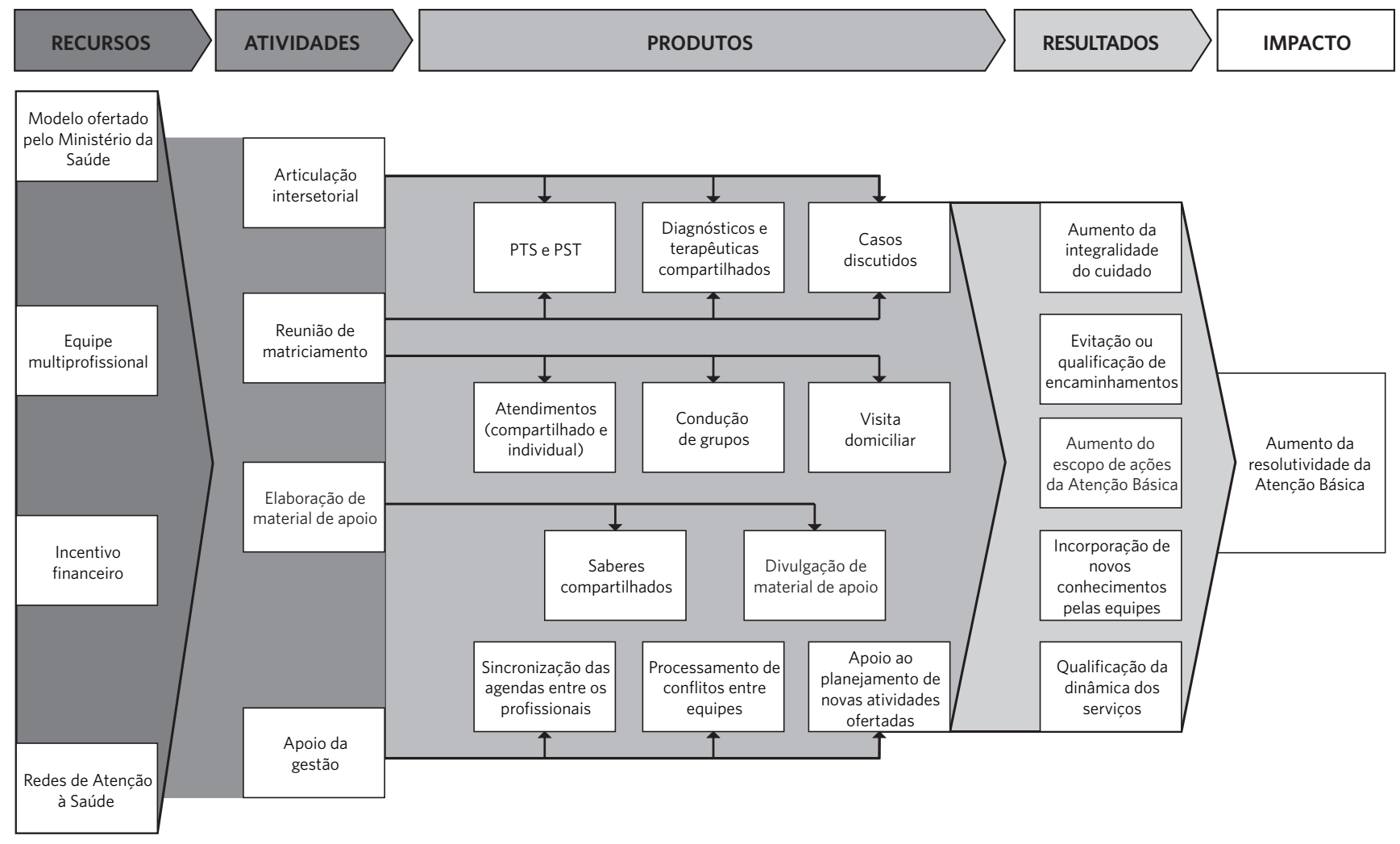

Fonte: Elaboração própria.

PTS: Projeto Terapêutico Singular; PST: Projeto de Saúde no Território.

Como recurso dessa iniciativa, há um modelo ofertado pelo MS, no qual os Nasf são divididos em três modalidades (a depender do número de equipes da $\mathrm{AB}$ a eles vinculadas e da somatória da carga horária dos profissionais que o integram), podendo ser compostos de partes não coincidentes de ampla lista de profissionais (8 especialidades médicas somadas a outras 11 profissões da saúde), articulados em 9 áreas consideradas estratégicas (atividade física ou práticas corporais; práticas integrativas e complementares; reabilitação; assistência farmacêutica; alimentação e nutrição; serviço social; saúde mental; saúde da mulher; e saúde da criança, do adolescente e do jovem) (BRASIL, 2012). Esses núcleos de apoio não constituem serviços com unidades físicas independentes e não são de livre acesso, seja para atendimento individual ou coletivo, devendo responder às demandas de equipes da RAS, da Academia da Saúde e mesmo de outras redes, como o Sistema Único de Assistência Social (Suas) (BRASIL, 2011), configurando-se como retaguarda especializada para estas mesmas equipes. O MS repassa aos municípios, fundo a fundo, incentivos em forma de recursos financeiros para a implantação (parcela única) e custeio mensal dos Nasf, cujos valores variam segundo o tipo de núcleo implantado (BRASIL, 2008).

Em sequência aos recursos, no modelo lógico, atividades e produtos dos Nasf se constituem a partir da compreensão de que essa iniciativa se dá pela articulação de profissionais de distintas áreas, que atuam de forma integrada junto às equipes da $\mathrm{AB}$, por meio de discussões de casos clínicos, atendimentos individuais e grupais 
compartilhados, de uma formulação conjunta de projetos terapêuticos individuais e territoriais, fomentando a intersetorialidade, com ações de prevenção, promoção e assistência em saúde (BRASIL, 2011).

As noções de apoio matricial e clínica ampliada, aliadas à proposta de RAS (CAMPOS; DOMITTI, 2007; CAMPOS; CUNHA; FIGUEIREDO, 2013) constituem, simultaneamente e de maneira atrelada, o referencial teórico e operacional dos Nasf.

Segundo a portaria ministerial que cria os Nasf (BRASIL, 2008), o objetivo desta iniciativa é ampliar as ações da $\mathrm{AB}$ no que tange ao seu escopo e à sua abrangência, bem como sua resolutividade, constituindo os resultados e impacto no modelo lógico.

\section{Questões oriundas dos grupos de interesse}

\section{ATUAC̣ÃO DOS PROFISSIONAIS E EQUIPES}

Boa parte da literatura considerada levanta questões surgidas a partir da implementação da proposta relativa aos Nasf. Foram encontradas análises do próprio referencial utilizado, enfocando a estratégia privilegiada (ANJOS ET AL., 2013; BARROS ET AL., 2015; MOURA; LUZIO, 2014; PASQUIM; ARRUDA, 2013; SAMPAIO ET AL., 2012), passando pela consideração das condições de trabalho dos profissionais (LANCMAN ET AL., 2013; LEITE; NASCIMENTO; OLIVEIRA, 2014) e suas atribuições (BONALDI E RIBEIRO, 2014; GOMES ET AL., 2014; GONÇALVES ET AL., 2015; HORI; NASCIMENTO, 2014; REIS ET AL., 2012), além de considerar a complexidade envolvida na colaboração entre os profissionais desse núcleo e os da EqSF (araújo; GALIMBERTTI, 2013 COSTA ET AL., 2014; FERRO ET AL., 2014; MATUDA ET AL., 2015; RIBEIRO ET AL., 2015).

Metade dos artigos considerados constituiu-se de dúvidas e buscas pelo estabelecimento das atribuições e dos papéis de cada uma das áreas integrantes da proposta, seja a fonoaudiologia (ANDRADE ET AL., 2014; COSTA ET AL., 2013; FERNANDES; NASCIMENTO; SOUSA, 2013;
MOLINI-AVEJONAS ET AL., 2014), a psicologia (AZEVEDO; KIND, 2013; CELA; OLIVEIRA, 2015; LEITE; ANDRADE; BOSI, 2013), a educação física (FALCl; BELISÁRIO, 2013; SANTOS ET AL., 2015; SOUZA; LOCH, 2011), a terapia ocupacional (LANCMAN; BARROS, 2011; REISA; VIEIRA, 2013), a fisioterapia (BARBOSA; FERREIRA; FURBINO, 2010; DIBAI FILHO; AVEIRO, 2012), a nutrição (AGUIAR; COSTA, 2015; RODRIGUES; BOSI, 2014) e a psiquiatria (VANNUCCHI; CARNEIRO JUNIOR, 2012). A ênfase no estabelecimento do que seriam os respectivos papéis destas categorias nos Nasf evidencia a carência de referenciais estabelecidos para dar sustentação às práticas profissionais nesse novo arranjo organizacional.

No entanto, a busca da identificação das funções de cada área profissional, nos Nasf, ao mesmo tempo em que evidencia limitações da proposta oficial para contemplar o que se espera de cada categoria, produz um paradoxo. Afinal, são buscadas respostas no espaço 'intra-área', desconsiderando-se o fato de que a iniciativa se baseia justamente em seu oposto, ou seja, na articulação interdisciplinar, que pressupõe colaboração entre diversos saberes e atuações profissionais. Buscam-se respostas no núcleo de competência e responsabilidade de cada profissão, quando as questões advêm justamente da necessidade de sua extrapolação, por meio de práticas em campos comuns de competência e responsabilidade (CAMPOS; DOMITTI, 2007). Some-se a isso o fato de que boa parte dos textos considera insuficiente a formação oferecida nas graduações, de modo a sustentar as práticas nos Nasf (AGUIAR; COSTA, 2015; AZEVEDO; KIND, 2013; BARBOSA ET AL., 2010; BONALDI; RIBEIRO, 2014; CELA; OLIVEIRA, 2015; FALCl; BELISÁRIO, 2013; FERRO ET AL., 2014; HORI; NASCIMENTO, 2014; LEITE; ANDRADE; BOSI, 2013; RODRIGUES; BOSI, 2014; SOUSA ET AL., 2015; SOUZA; LOCH, 2011), indicando que, no interior das áreas, parece não haver respostas estabelecidas. Discussões que avançam para além de uma dada categoria ainda são pouco frequentes, como aquela feita por Costa et al. (2014).

Especificamente em relação aos recursos requeridos para a atuação dos núcleos, apontam-se, além de um elevado número de 
equipes para matriciar - o que compromete o tempo necessário para um efetivo apoio -, a precariedade das condições de trabalho representada pela falta de espaço físico e de materiais, de aparatos tecnológicos, de meios de comunicação e de transporte, para o exercício das ações do Nasf (AGUIAR; COSTA, 2015; ANDRADE ET AL., 2014; BONALDI; RIBEIRO, 2014; COSTA ET AL., 2013; COSTA ET AL., 2014; FALCI; BELISÁRIO, 2013; GONC̣ALVES ET AL., 2015; HORI; NASCIMENTO, 2014; LANCMAN; BARROS, 2011; LANCMAN ET AL., 2013; LEITE; NASCIMENTO; OLIVEIRA, 2014; MOLINI-AVEJONAS ET AL., 2014; REISA; VIEIRA, 2013; SOUSA ET AL., 2015; SOUZA; LOCH, 2011; VANNUCCHI; CARNEIRO JUNIOR, 2012). Estas questões são corroboradas por outros trabalhos, que abordam de maneira específica a sobrecarga de trabalho (ARAÚJO; GALIMBERTTI, 2013; AZEVEDO; KIND, 2013; BONALDI; RIBEIRO, 2014; LANCMAN; BARROS, 2011; LEITE; ANDRADE; BOSI, 2013; LEITE; NASCIMENTO; OLVEIRA, 2014; VANNUCCHI; CARNEIRO JUNIOR, 2012), a quantidade e diversidade de atribuições (FERRO ET AL., 2014; SOUSA ET AL., 2015) e a demanda reprimida (BARBOSA ET AL., 2010; BONALDI; RIBEIRO, 2014; LANCMAN; BARROS, 2011; REISA; VIEIRA, 2013). Além disso, a instabilidade do vínculo empregatício dos profissionais do Nasf, contratados por meio de organizações sociais, ocasiona diferenças nas cargas horárias e aumento na rotatividade, comprometendo a consolidação da proposta (LANCMAN; BARROS, 2011; PASQUIM; ARRUDA, 2013; REISA; VIEIRA, 2013), o que vem somar-se a problemas oriundos de equipes incompletas (BONALDI; RIBEIRO, 2014; REISA; VIEIRA, 2013; COSTA ET AL., 2014; HORI; NASCIMENTO, 2014), à falta de respaldo organizacional (CELA; OLIVEIRA, 2015; HORI; NASCIMENTO, 2014; LEITE; ANDRADE; BOSI, 2013; SAMPAIO ET AL., 2012; SOUSA; OLIVEIRA; COSTA, 2015) e à submissão dos Nasf às metas estabelecidas originalmente para as EqSF (MATUdA ET AL., 2015). Salienta-se que a proposta de apoio matricial, central aos Nasf, supera os aspectos técnicos, uma vez que pressupõe avanços em relação aos desníveis de poder entre equipes e categorias profissionais, podendo suscitar questões não comumente consideradas no interior das equipes (BARROS ET AL., 2015; REISA; VIEIRA, 2013; RIBEIRO ET AL., 2015).

\section{SOBRE O MODELO TECNOASSISTENCIAL DOS NASF}

Os documentos oficiais de orientação são considerados genéricos (LANCMAN; BARROS, 2011; SAMPAIO ET AL., 2012), e os prazos para suas edições não foram precisos, uma vez que o descompasso entre a Portaria $\mathrm{n}^{\circ} 154$ (BRASIL, 2008) e a publicação do 'Caderno de Atenção Básica no 27’ (BRASIL, 2009) favoreceu a criação de diferentes modelos de Nasf, segundo os diferentes contextos político-institucionais nos municípios nos quais foram implantados, gerando significativas diferenças entre núcleos, até no mesmo município. Para alguns autores, haveria insuficiente direcionamento político e tecnoassistencial na indução da implantação de Nasf pelo MS, resultando em diferentes processos de trabalho, e definição das ações com base estritamente empírica ou contingente (ARAÚJO; GALIMBERTTI, 2013; AZEVEDO; KIND, 2013; HORI; NASCIMENTO, 2014; LANCMAN ET AL., 2013; RODRIGUES; BOSI, 2014).

A organização do trabalho do Nasf se daria, essencialmente, nas reuniões de matriciamento da EqSF (BARROS ET AL., 2015; FERNANDES; NASCIMENTO, SOUSA, 2013; GONÇALVES ET AL., 2015; LANCMAN ET AL., 2013; MOLINI-AVEJONAS ET AL., 2014; MOURA; LUZIO, 2014). No entanto, é relevante que esses encontros sejam considerados fundamentais e, ao mesmo tempo, especialmente desafiadores, dada a dificuldade de articulação entre profissionais da EqSF e do Nasf, mesmo no contexto das RAS (ARAúJo; GALIMBERTTI, 2013; AZEVEDO; KIND, 2013; BARBOSA ET AL., 2010; BONALDI; RIBEIRO, 2014; CELA; OLIVEIRA, 2015; COSTA ET AL., 2013; COSTA ET AL., 2014; DIBAI FILHO; AVEIRO, 2012; FALCI; BELISÁRIO, 2013; FERRO ET AL., 2014; GONÇALVES ET AL., 2015; HORI; NASCIMENTO, 2014; LANCMAN; BARROS, 2011; LANCMAN ET AL., 2013; LEITE; NASCIMENTO; OLIVEIRA, 2014; PASQUIM; ARRUDA, 2013; REISA; VIEIRA, 2013; RIBEIRO ET AL., 2015; RODRIGUES; BOSI, 2014; SOUZA; LOCH, 2011; VANNUCCHI; CARNEIRO JUNIOR, 2012), sendo que as práticas intersetoriais são consideradas restritas ou mesmo inexistentes (AGUIAR; COSTA, 2015; ANJOS ET AL., 2013; BONALDI; RIBEIRO, 2014; CELA; OLIVEIRA, 2015; FERRO ET AL., 2014; HORI; NASCIMENTO, 2014; 
LEITE; ANDRADE; BOSI, 2013; PASQUIM; ARRUDA, 2013).

Articulada ao fato, já considerado, de que metade dos artigos se ocupa da busca de respostas sobre a atuação das profissões, identifica-se a transposição, para o interior do Nasf, do exercício de atividades típicas das áreas profissionais que o compõem, como o atendimento clínico individual. Esta situação ocorre como forma de responder às expectativas iniciais da EqSF, à grande demanda existente por atendimentos especializados ou à solicitação direta dos próprios usuários (AGUIAR; COSTA, 2015; ARAÚJO; GALIMBERTTI, 2013; AZEVEDO; KIND, 2013; BARBOSA ET AL., 2010; COSTA ET AL., 2013; DIBAI FILHO; AVEIRO, 2012; FERRO ET AL., 2014; GOMES ET AL., 2014; GONÇALVES ET AL., 2015; LANCMAN ET AL., 2013; LEITE; ANDRADE; BOSI, 2013; MOLINI-AVEJONAS ET AL., 2014; PASQUIM; ARRUDA, 2013; RODRIGUES; BOSI, 2014; SANTOS ET AL., 2015; SOUSA ET AL., 2015). Além do atendimento especializado individual, outras ações caras à proposta acontecem, como a atenção domiciliar conjunta (AZEVEDO; KIND, 2013; DIBAI FILHO; AVEIRO, 2012; GONÇALVES ET AL., 2015; LEITE ET AL., 2013; MOLINI-AVEJONAS ET AL., 2014; REIS ET AL., 2012; SOUSA ET AL., 2015; SOUZA; LOCH, 2011) e o trabalho com grupos (AZEVEDO; KIND, 2013; BONALDI; RIBEIRO, 2014; DIBAI FILHO; AVEIRO, 2012; GOMES ET AL., 2014; GONÇALVES ET AL., 2015; LEITE; ANDRADE; BOSI, 2013; MOURA; LUZIO, 2014; SOUSA; OLIVEIRA; COSTA, 2015), que constituem atividades ofertadas com maior frequência por esses núcleos.

A educação permanente se faz presente através de oficinas de educação em saúde (BONALDI; RIBEIRO, 2014; COSTA ET AL., 2013; SOUZA; LOCH, 2011) e capacitação para os agentes comunitários de saúde (LEITE; ANDRADE; BOSI, 2013; MOLINIAVEjonAs et Al., 2014). Porém, diferentemente do que se concebeu na elaboração dos Nasf, o potencial da discussão de casos ou dos atendimentos compartilhados, para troca de saberes entre os profissionais e incorporação de novos conhecimentos pelas equipes, raramente é considerado nos documentos analisados como estratégia de formação (FERNANDES; NASCIMENTO; SOUSA, 2013; LANCMAN ET AL., 2013; MATUDA ET AL., 2015; MOURA; LUZIO, 2014).

Um trabalho mais resolutivo e a ampliação do escopo de ações ofertadas foram mencionados como consequências do trabalho de uma equipe multiprofissional e do matriciamento (BARROS ET AL., 2015; COSTA ET AL., 2014; LEITE; MASCIMENTO; OLIVEIRA, 2014; REIS ET AL., 2012). O Nasf foi tido como uma proposta interessante para cumprir a função de apoio matricial e para a gestão integrada do cuidado (MOURA; LUZIO, 2014; REIS ET AL., 2012), no entanto, a maioria dos estudos aponta a necessidade de avanços na atuação dos núcleos, como melhoria da qualificação de seus profissionais (ANJOS ET AL., 2013; BARBOSA ET AL., 2010; CELA; OLIVEIRA, 2015; FALCl; BELISÁRIO, 2013; FERNANDES; NASCIMENTO; SOUSA, 2013; HORI; NASCIMENTO, 2014; LANCMAN ET AL., 2013; LEITE; ANDRADE; BOSI, 2013; MATUDA ET AL., 2015; RODRIGUES; BOSI, 2014; SANTOS ET AL., 2015), revisão de seus processos de trabalho e melhoria da estrutura (ANDRADE ET AL., 2014; ANJOS ET AL., 2013; HORI; NASCIMENTO, 2014; LANCMAN ET AL., 2013; LEITE ET AL., 2014; MATUDA ET AL., 2015; MOLINI-AVEJONAS ET AL., 2014), permanecendo, assim, a necessidade de enfrentamento a antigos desafios da saúde pública (PASQUIM; ARRUDA, 2013).

\section{Subsídios a processos avaliativos dos Nasf}

Para Champagne et al. (2011A), o caráter avaliável de uma intervenção é aferido pelo grau em que quatro condições são observadas: 1) a possibilidade de apresentar a iniciativa na forma de um modelo; 2) coerência e adequação do modelo aos objetivos; 3) suficiência dos recursos para o alcance dos objetivos; e 4) interesse manifesto, dos agentes, pela avaliação. Tomando como base estas condições, é possível afirmar que a proposta de Nasf pode ser articulada e formalizada graficamente, há coerência entre modelo e objetivos, e a quantidade de questões levantadas nos artigos considerados indicam, ao menos entre esses autores, questões que podem ser contempladas por meio de processos avaliativos. A suficiência dos recursos fugiria à nossa capacidade de resposta, dada a amplitude geográfica e numérica da iniciativa aqui enfocada. 
No entanto, as afirmativas feitas no parágrafo anterior se sustentam, sobretudo, calcadas nas proposições e nos documentos oriundos do MS. Dificilmente, tais proposições sistematizadas na figura 1 espelhariam o conjunto das práticas em torno dos Nasf, no Brasil. A julgar pelo debate identificado na literatura analisada, haveria dissensos, tanto sobre a concepção quanto em relação às maneiras mais adequadas de operacionalizar a proposta. Deve-se notar, conforme desenvolvido no tópico 'Sobre o modelo tecnoassistencial dos Nasf', que estratégias essenciais à proposta são justamente aquelas consideradas especialmente difíceis, como a colaboração entre profissionais, com as EqSF e o trabalho intersetorial. Identificouse que processos avaliativos poderão privilegiar tanto a análise estratégica quanto a análise lógica, propostas por Champagne et al. (2011B). Para os autores, as análises lógica e estratégica consideram, entre outras coisas, a pertinência das parcerias estratégicas feitas para a implementação de uma iniciativa complexa, como os Nasf, além de considerar as hipóteses teóricas que dão base à ação, e os meios requeridos. Pode-se compreender a pergunta de cada categoria sobre seu respectivo papel no Nasf como uma indagação sobre a própria estratégia e lógica da proposta que sustenta esses núcleos, o que poderia ser efetivamente considerado, em se tratando de futuros processos avaliativos, por meio das análises estratégica e lógica desenvolvidas pelos autores citados (CHAMPAGNE ET AL., 2011B). Ou seja, a existência de questões ainda muito essenciais à proposta, dirigidas à efetiva função dos Nasf - modo de operar, formas de colaboração entre profissionais e áreas, entre outras - apontam que, mais do que enfocar resultados, efeitos e impactos, esses núcleos carecem de estudos avaliativos que abordem aspectos ligados à sua concepção e estratégias.

Além das questões relativas ao modelo tecnoassistencial, abordáveis por meio de análises estratégicas e lógicas em processos avaliativos, da literatura revisada emergem outras questões que se localizam, sobretudo, no âmbito do conjunto de 'atividades' e 'produtos' integrantes do modelo lógico (figura 1). Ali podem ser inseridas questões relativas às dificuldades de articulação com outros setores, para além da Saúde; eventuais entraves à elaboração e ao acompanhamento de Projetos Terapêuticos Singulares (PTS); e o compartilhamento de saberes entre profissionais de distintas categorias. Inquietações reiteradas nos artigos e relativas à qualificação profissional, ao processo de trabalho e à estrutura ofertada poderiam ser abordadas por meio de estudos que enfocassem a avaliação de qualidade (NEMES, 2001).

\section{Conclusões}

Os Nasf se sustentam sobre um conjunto de proposições, recomendações e direcionamentos provenientes do MS suficientes para o estabelecimento claro e articulado da proposta por meio de um modelo lógico. No entanto, o encontro do que foi concebido no plano central e as muitas e diversas realidades político-institucionais, nas várias regiões do País, geram descompassos de distintas naturezas. Mas, é justamente no descompasso entre o planejado e o implementado que se justifica boa parte das avaliações. Algumas questões oriundas da implementação da proposta parecem colocar em questão concepções e hipóteses presentes no arcabouço teórico que sustenta a iniciativa. Mais do que em outros programas, eventuais avaliações dos Nasf devem considerar seus próprios pressupostos, lançando mão das análises estratégicas e lógicas citadas.

Há também um conjunto importante de questões, presente nos artigos considerados e inserido, sobretudo, no que se refere às atividades e aos produtos desses núcleos, em detrimento dos chamados efeitos ou resultados. Esse fato parece reforçar que as questões que se colocam para a avaliação 
situam-se preponderantemente nos aspectos ligados à concepção e ao funcionamento da proposta, sendo ainda precoces indagações dirigidas aos resultados e impactos, visto que os passos anteriores permanecem, ainda, como alvos de questionamentos e indagações, o que parece justificar que avaliações do grau de implementação dos Nasf, em distintas regiões, se afigurem como oportunas e pertinentes.

Finalmente, considerou-se que o estudo de avaliabilidade dos Nasf permitiu uma visão de conjunto da proposta, sua modelização e definição das principais questões que a atravessam, tanto do ponto de vista de sua concepção quanto de suas práticas. A utilização de revisão bibliográfica como meio para a obtenção das principais questões levantadas, por parte dos agentes envolvidos, se mostrou suficiente para a realização deste estudo.

\section{Colaboradores}

Patrícia Caroline Iacabo Correia participou da concepção do estudo, definição do desenho metodológico, revisão de literatura, discussão dos resultados, redação e revisão crítica do texto. Patrícia Martins Goulart participou da revisão de literatura. Juarez Pereira Furtado participou da concepção do estudo, definição do desenho metodológico, redação e revisão crítica do texto.

\section{Agradecimentos}

Agradecimentos à Professora Patrícia Coelho de Soárez, pelo apoio na qualificação do percurso da revisão de literatura, e ao Wagner Yoshizaki Oda, pelo fornecimento de sua ideia para a sistematização dos dados.

\section{Referências}

AGUIAR, C. B.; COSTA, N. M. S. C. Formação e atuação de nutricionistas dos Núcleos de Apoio à Saúde da Família. Rev. Nutr., Campinas, v. 28, n. 2, p. 207-216, abr. 2015.

ANDRADE, A. F. et al. Avaliação das ações da Fonoaudiologia no NASF da cidade do Recife. Audiol. Commun. Res., São Paulo, v. 19, n. 1, p. 52-60, mar. 2014.

ANJOS, K. F. Perspectivas e desafios do núcleo de apoio à saúde da família quanto às práticas em saúde. Saúde em Debate, Rio de Janeiro, v. 37, n. 99, p. 672-680, dez. 2013. colaboração interprofissional na estratégia saúde da família. Psicol. Soc., Belo Horizonte, v. 25, n. 2, p. 461468, 2013.

AZEVEDO, N. S.; KIND, L. Psicologia nos núcleos de apoio à saúde da família em Belo Horizonte. Psicol. Ciênc. Prof., Brasília, DF, v. 33, n. 3, p. 520-535, 2013.

BARBOSA, E. G.; FERREIRA, D. L. S.; FURBINO, S. A. R. Experiência da fisioterapia no Núcleo de Apoio à Saúde da Família em Governador Valadares, MG. Fisioter. Mov. (Impr.), Curitiba, v. 23, n. 2, p. 323-330, jun. 2010.

BARROS, J. O. et al. Estratégia do apoio matricial: 
a experiência de duas equipes do Núcleo de Apoio à Saúde da Família (NASF) da cidade de São Paulo, Brasil. Ciênc. Saúde Coletiva, Rio de Janeiro, v. 20, n. 9, p. 2847-2856, set. 2015.

BONALDI, A. P.; RIBEIRO, M. D. Núcleo de apoio à saúde da família: as ações de promoção da saúde no cenário da Estratégia Saúde da Família. Rev. APS, Juiz de Fora, v. 17, n. 2, p. 195-203, abr./jun. 2014.

BRASIL. Ministério da Saúde. Gabinete do Ministro. Portaria MS/GM n ${ }^{\circ} 154$, de 24 de janeiro de 2008. Cria os Núcleos de Apoio à Saúde da Família - NASF. Diário Oficial [da] União. Brasília, DF, 24 jan. 2008. Disponível em: <http://bvsms.saude.gov.br/bvs/saudelegis/ gm/2008/prt0154_24_01_2008.html>. Acesso em: 19 jan. 2017.

Ministério da Saúde. Gabinete do Ministro. Portaria MS/GM n ${ }^{\circ} 2488$, de 21 de outubro de 2011. Aprova a Política Nacional de Atenção Básica, estabelecendo a revisão de diretrizes e normas para a organização da Atenção Básica, para a Estratégia Saúde da Família (ESF) e o Programa de Agentes Comunitários de Saúde (PACS). Diário Oficial [da] União. Brasília, DF, 21 out. 2011. Disponível em: <http://bvsms.saude.gov.br/bvs/saudelegis/gm/2011/ prt2488_21_10_2011.html>. Acesso em: 19 jan. 2017.

Ministério da Saúde. Gabinete do Ministro. Portaria MS/GM no 3124, de 28 de dezembro de 2012. Redefine os parâmetros de vinculação dos Núcleos de Apoio à Saúde da Família (NASF) Modalidades 1 e 2 às Equipes Saúde da Família e/ou Equipes de Atenção Básica para populações específicas, cria a Modalidade NASF 3, e dá outras providências. Diário Oficial [da] União. Brasília, DF, 28 dez. 2012. Disponível em: <http://bvsms.saude.gov.br/bvs/saudelegis/gm/2012/ prt3124_28_12_2012.html>. Acesso em: 19 jan. 2017.

Ministério da Saúde. Secretaria de Atenção à Saúde. Diretrizes do NASF. Brasília, DF: MS, 2009. (Cadernos de Atenção Básica, n. 27).

Ministério da Saúde. Secretaria de Atenção à Saúde. Núcleo de Apoio à Saúde da Família. Brasília, DF: MS, 2014. (Cadernos de Atenção Básica, n. 39).
CAMPOS, G. W. S.; CUNHA, G. T.; FIGUEIREDO, M. D. Práxis e Formação Paideia: apoio e co-gestão em saúde. São Paulo: Hucitec, 2013.

CAMPOS, G. W. S.; DOMITTI, A. C. Apoio matricial e equipe de referência: uma metodologia para gestão do trabalho interdisciplinar em saúde. Cad. Saúde Pública, Rio de Janeiro, v. 23, n. 2, p. 399-407, fev. 2007.

CELA, M.; OLIVEIRA, I. F. O psicólogo no Núcleo de Apoio à saúde da Família: articulação de saberes e ações. Estud. Psicol., Natal, v. 20, n. 1, p. 31-39, mar. 2015.

CHAMPAGNE, F. et al. A análise lógica. In: BROUSSELLE, A. et al. (Org.). A avaliação: conceitos e métodos. Rio de Janeiro: Fiocruz, 2011a.

CHAMPAGNE, F. et al. Modelizar as Intervenções. In: In: BROUSSELLE, A. et al. (Org.). A avaliação: conceitos e métodos. Rio de Janeiro: Fiocruz, 2011b.

COSTA, L. S. et al. A prática do fonoaudiólogo nos Núcleos de Apoio à Saúde da Família em municípios paraibanos. CoDAS, São Paulo, v. 25, n. 4, p. 381-387, 2013

COSTA, J. P. et al. Resolubilidade do cuidado na atenção primária: articulação multiprofissional e rede de serviços. Saúde em Debate, Rio de Janeiro, v. 38, n. 103, p. 733-743, dez. 2014.

DIBAI FILHO, A. V.; AVEIRO, M. C. Atuação dos fisioterapeutas dos Núcleos de Apoio à Saúde da Família entre idosos do município de Arapiraca - AL, Brasil. Rev. Bras. Promoç. Saúde, Fortaleza, v. 25, n. 4, p. 397-404, out./dez., 2012.

FALCI, D. M.; BELISARIO, S. A. A inserção do profissional de educação física na atenção primária à saúde e os desafios em sua formação. Interface (Botucatu), Botucatu, v. 17, n. 47, p. 885-899, dez. 2013.

FERNANDES, T. L.; NASCIMENTO, C. M. B.; SOUSA, F. O. S. Análise das atribuições dos fonoaudiólogos do NASF em municípios da região metropolitana de Recife. Rev. CEFAC, Campinas, v. 15, n. 1, p. 153-159, 2013 
FERRO, L. F. et al. Interdisciplinaridade e intersetorialidade na Estratégia Saúde da Família e no Núcleo de Apoio à Saúde da Família: potencialidades e desafios. O Mundo da Saúde, São Paulo, v. 38, n. 2, p. 129-138, 2014

FURTADO, J. P.; ONOCKO-CAMPOS, R. A transposição das políticas de saúde mental no Brasil para a prática nos novos serviços. Revista Latinoamericana de Psicopatologia Fundamental, São Paulo, v. 3, n. 1, 2005.

FURTADO, J. P. Avaliação de programas e serviços em saúde. In: MINAYO, M. C. et al. (Org.). Tratado de Saúde Coletiva. 2. ed. São Paulo: Hucitec, p. 221-246, 2012.

GOMES, G. A. O. et al. Characteristics of physical activity programs in the Brazilian primary health care system. Cad. Saúde Pública, Rio de Janeiro, v. 30, n. 10, p. 2155-2168, out. 2014.

GONCALVES, R. M. A. et al. Estudo do trabalho em Núcleos de Apoio à Saúde da Família (NASF), São Paulo, Brasil. Rev. Bras. Saúde Ocup., São Paulo, v. 40, n. 131, p. 59-74, jun. 2015.

HIGGINS, J. P. T.; GREEN, S. (editors). Cochrane Handbook for Systematic Reviews of Interventions, Version 5.1.0 [updated March 2011]. The Cochrane Collaboration, 2011. Disponível em: <http://handbook. cochrane.org>. Acesso em: 4 jan. 2016.

HORI, A. A.; NASCIMENTO, A. F. O Projeto Terapêutico Singular e as práticas de saúde mental nos Núcleos de Apoio à Saúde da Família (NASF) em Guarulhos (SP), Brasil. Ciência \&t Saúde Coletiva, Rio de Janeiro, v. 19, n. 8, p. 3561-3571, ago. 2014.

LANCMAN, S.; BARROS, J. O. Estratégia de saúde da família (ESF), Núcleo de Apoio à Saúde da Família (NASF) e terapia ocupacional: problematizando as interfaces. Rev. Ter. Ocup. Univ. São Paulo, São Paulo, v. 22, n. 3, p. 263-269, set./dez. 2011.

LANCMAN, S. et al. Estudo do trabalho e do trabalhar no Núcleo de Apoio à Saúde da Família. Rev. Saúde Pública, São Paulo, v. 47, n. 5, p. 968-975, 2013.
LEITE, D. C.; ANDRADE, A. B.; BOSI, M. L. M. A inserção da Psicologia nos Núcleos de Apoio à Saúde da Família. Physis, Rio de Janeiro, v. 23, n. 4, p. 1167-1187, dez. 2013.

LEITE, D. F.; NASCIMENTO, D. D. G.; OLIVEIRA, M. A. C. Qualidade de vida no trabalho de profissionais do NASF no município de São Paulo. Physis, Rio de Janeiro, v. 24, n. 2, p. 507-525, 2014.

LEVITON, L. C. et al. Evaluability assessment to improve public health policies, programs and practices. Annu. Rev. Public. Health, Palo Alto, v. 31, p. 213-233, 2010.

MACHADO, C. V. et al. Federalismo e política de saúde: comissões intergovernamentais no Brasil. Rev. Saúde Pública USP, v. 48, n. 4, p. 642-650, 2014.

MATUDA, C. G. et al. Colaboração interprofissional na Estratégia Saúde da Família: implicações para a produção do cuidado e a gestão do trabalho. Ciência \& Saúde Coletiva, Rio de Janeiro, v. 20, n. 8, p. 2511-2521, ago. 2015.

MEDEIROS, P. F. P. et al. Um estudo sobre a avaliabilidade do Programa + Vida: política de redução de danos em álcool, fumo e outras drogas do município de Recife, Brasil. Rev. Bras. Saúde Mater. Infant., Recife, v. 10, supl. 1, p. s209-s217, nov. 2010.

MENDES, M. F. M.; BEZERRA, G. C. L. A.; DUBEUX, L. S. Avaliabilidade ou pré-avaliação de um programa. In: SAMICO, I. et al. (Org.). Avaliação em saúde - bases conceituais e operacionais, Recife: IMIP, 2010.

MOLINI-AVEJONAS, D. R. et al. Inserção e atuação da Fonoaudiologia nos Núcleos de Apoio à Saúde da Família. CoDAS, São Paulo, v. 26, n. 2, p. 148-154, abr. 2014.

MOURA, R. H.; LUZIO, C. A. O apoio institucional como uma das faces da função apoio no Núcleo de Apoio à Saúde da Família (NASF): para além das diretrizes. Interface (Botucatu), Botucatu, v. 18, n. 1, p. 957-970, 2014 
NEMES, M. I. B. Avaliação em saúde: questões para os programas de DST/AIDS no Brasil. Rio de Janeiro: ABIA, 2001. (Coleção ABIA. Fundamentos de Avaliação, n. 1).

OLIVEIRA, C. M. et al. Avaliabilidade do Programa de Valorização do Profissional da Atenção Básica (PROVAB): desafios para gestão do trabalho. Ciência \&t Saúde Coletiva, Rio de Janeiro, v. 20, n. 10, p. 2999-3010, out. 2015.

PADILHA, M. A.; OLIVEIRA, C. M.; FIGUEIRO, A

C. Estudo de avaliabilidade do Programa Academia Carioca da Saúde: desafios para a promoção da saúde. Saúde em Debate, Rio de Janeiro, v. 39, n. 105, p. 375386, jun. 2015.

PASQUIM, H. M.; ARRUDA, M. S. B. Núcleo de apoio à saúde da família: Revisão narrativa sobre o apoio Matricial na atenção básica. Corpus et Scientia, Rio de Janeiro, v. 9, n. 2, p. 34-44, jul./dez. 2013.

REIS, D. C. et al. Perfil de atendimento de um Núcleo de Apoio à Saúde da Família na área de reabilitação, Município de Belo Horizonte, Estado de Minas Gerais, Brasil, 2009. Epidemiol. Serv. Saúde, Brasília, DF, v. 21, n. 4, p. 663-674, dez. 2012.

REISA, F.; VIEIRA, A. C. V. C. Perspectivas dos terapeutas ocupacionais sobre sua inserção nos Núcleos de Apoio à Saúde da Família (NASF) de Fortaleza, CE. Cad. Ter. Ocup. UFSCar, São Carlos, v. 21, n. 2, p. 351-360, 2013.

RIBEIRO, H. M. C. B. et al. Representações sociais de profissionais de núcleos de apoio à saúde da família sobre interdisciplinaridade. Trabalho, Educação e Saúde. Rio de Janeiro, v. 13, n. 2, p. 97-115, 2015.

RODRIGUES, D. C. M.; BOSI, M. L. M. O lugar do nutricionista nos Núcleos de Apoio à Saúde da Família. Rev. Nutr., Campinas, v. 27, n. 6, p. 735-746, dez. 2014.

SAMPAIO, J. et al. O Nasf como dispositivo de gestão: limites e possibilidades. Revista Brasileira de Ciências da Saúde, João Pessoa, v. 16, n. 3, p. 317-324, 2012.
SANTANA, C. L. A. A integração da Saúde Mental na Estratégia Saúde da Família. Interface (Botucatu), Botucatu, v. 18, n. 51, p. 797-798, dez. 2014.

SANTOS, S. F. S. et al. The work of physical education professionals in Family Health Support Centers (NASF): a national survey. Rev. Bras. Cineantropom. Desempenho Hum., Florianópolis, v. 17, n. 6, p. 693-703, dez. 2015.

SILVA, M. A. P. et al. Atenção Básica em Alagoas: expansão da Estratégia Saúde da Família, do Nasf e do componente alimentação/nutrição. Saúde em Debate, Rio de Janeiro, v. 38, n. 103, p. 720-732, dez. 2014.

SOUZA, S. C.; LOCH, M. R. Intervenção do profissional de educação física nos Núcleos de Apoio à Saúde da Família em municípios do norte do Paraná. Revista Brasileira de Atividade Física \&t Saúde, Pelotas, v. 16, n. $1,2011$.

SOUSA, D.; OLIVEIRA, I. F.; COSTA, A. L. F. Entre o especialismo e o apoio: psicólogos no Núcleo de Apoio à Saúde da Família. Psicologia USP, São Paulo, v. 26, n. 3 , p. $474-483,2015$.

SUCHMAN, E. R. Evaluative research: principles and practice in public service and social action programs. New York: Russel Sage Foundation, 1967.

VANNUCCHI, A. M. C.; CARNEIRO JUNIOR, N.

Modelos tecnoassistenciais e atuação do psiquiatra no campo da atenção primária à saúde no contexto atual do Sistema Único de Saúde, Brasil. Physis, Rio de Janeiro, v. 22, n. 3, p. 963-982, 2012.

WHOLEY, J. S. Evaluability assessment: developing program theory. New Directions for Program Evaluation, Hoboken, v. 33, p. 77-92, 1987.

Recebido para publicação em abril de 2016

Versão final em julho de 2016

Conflito de interesses: inexistente

Suporte financeiro: não houve 